\title{
Teoria dos Campos Conceituais na análise de programação em Scratch
}

Kátia Coelho da Rocha - PPGEMat/UFRGS - katiacoelhorocha@ gmail.com Marcus Vinicius de Azevedo Basso - PPGEMat/UFRGS - mbasso@ufrgs.br

Resumo A compreensão da construção do conceito de ângulo via atividades de programação em Scratch foi a base de uma pesquisa de mestrado que originou este artigo. Nessa pesquisa, de caráter qualitativo realizada com dezesseis estudantes do sexto ano do ensino fundamental de uma escola pública localizada na grande Porto Alegre, a Teoria dos Campos Conceituais de Gerard Vergnaud foi utilizada na concepção e análise de atividades de programação em Scratch. Concebida como uma teoria que tem como foco o processo de aprendizagem e, particularmente, a aprendizagem de matemática no contexto escolar, como resultado e contribuição deste estudo, destaca-se o papel da Teoria dos Campos Conceituais na interpretação dos esquemas utilizados pelos estudantes em atividade de programação.

Palavras-chave: teoria dos campos conceituais, scratch, aprendizagem de matemática, esquema

\section{Theory of Conceptual Fields in Programming Analysis in Scratch}

Abstract The comprehension of the construction of angle concept through programming activities via Scratch was the basis of a master's research that originated this article. In this research, of qualitative character, carried out with 16 students from 6th grade of elementary school in a public school in Porto Alegre, it was used The Theory of the Conceptual Fields from Gerard Vergnaud, for the conception and analyses of Scratch programming activities. Planned as a theory whom has the focus on the learning process and, particularly, the learning of math in the scholar context, as a result and contribution of this study, we highlight the role of the Theory of the Conceptual Fields in the interpretation of the schemas used by the students in programming activities.

Keywords: theory of conceptual fields, scratch, learning of math, schema

\section{INTRODUÇÃO}

O destaque dado às práticas de programação na escola tem evidenciado a importância do desenvolvimento do pensamento computacional cada vez mais cedo entre crianças e adolescentes. O pensamento computacional defendido por Wing (2006), propõe a utilização de diferentes estratégias de pensamento que levem o estudante a pensar por procedimentos (PAPERT, 1985; VITALLE, 1991; WING, 2006).

Uma das ferramentas que pretende auxiliar no desenvolvimento desse tipo de pensamento é o Scratch, uma linguagem de programação em blocos desenvolvida pelo Massachusetts Institute of Technology (RESNICK et all, 2009). Ao utilizar essa ferramenta durante a pesquisa de mestrado com estudantes do sexto ano do ensino fundamental, identificamos que ela permite a compreensão do conceito de ângulo entre outros conceitos matemáticos (ROCHA, 2017). A programação de situações variadas em Scratch permite ao estudante testar, confrontar, combinar e recombinar seus conceitos e teoremas-em-ação (VERGNAUD, 1993).

Apresentamos neste artigo o uso da Teoria dos Campos Conceituais como referencial teórico para a análise de atividades de programação em Scratch. Destacamos nas seções seguintes a interface do Scratch, sua proposta de programação 
em blocos e os principais elementos da teoria de Gerard Vergnaud relacionando-os com possíveis atividades de programação no software.

\section{O SOFTWARE SCRATCH}

O Scratch é um software criado para o desenvolvimento do pensamento computacional. Sua estrutura está baseada na linguagem LOGO criada por Seymour Papert na década de 70. A programação se estrutura em blocos permitindo a criação de comandos a partir do encaixe desses blocos pré-formatados. A figura 1 apresenta a tela inicial do Scratch e suas áreas principais

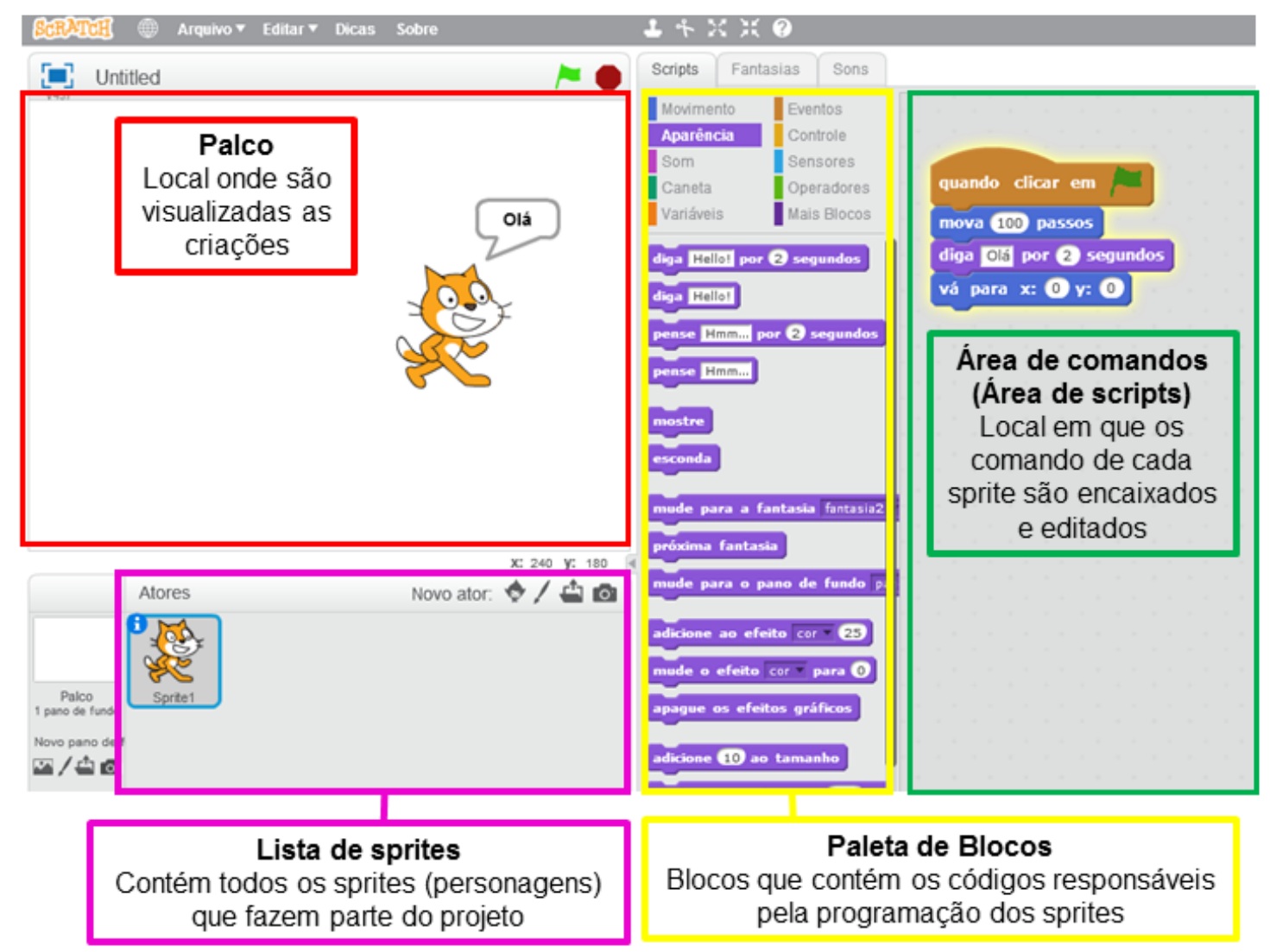

Figura 1 - Interface do Scratch

Fonte: acervo da primeira autora

A área denominada "palco" contém um plano cartesiano em que a origem corresponde ao centro do palco. O palco contém um sprite (ator) que executa a ação programada na área de comandos.

A programação é realizada através dos blocos que são encaixados para formar o código, conforme pode ser observado na figura 2. Esses blocos já estão prontos, não havendo preocupação com a escrita dos códigos, tornando a linguagem visual e acessível. As ações dos blocos são executadas na sequência em que foram encaixados. 


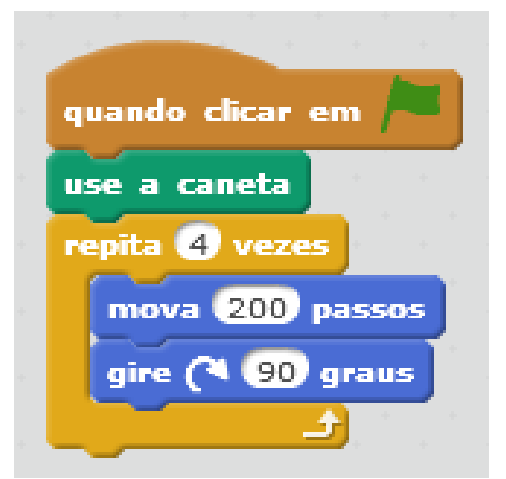

Figura 2 - Comando para desenhar um quadrado Fonte: acervo da primeira autora

Nas seções seguintes apresentamos programas em Scratch e possíveis interpretações de esquemas de pensamento via Teoria dos Campos Conceituais.

\section{A TEORIA DOS CAMPOS CONCEITUAIS E SUAS INFLUENCIAS}

A Teoria dos Campos Conceituais foi criada por Gérard Vergnaud, um matemático, psicólogo e filósofo francês. Aluno de Jean Piaget em Genebra, atualmente é diretor emérito de estudos do Centro Nacional de Pesquisas Científicas (CNRS) em Paris.

Ao desenvolver sua teoria, Vergnaud demonstra interesse pelo processo de ensino e aprendizagem da matemática no contexto escolar, investigando como o estudante aprende em ação. Desta forma, a Teoria dos Campos Conceituais foi inicialmente desenvolvida para explicar o processo de construção de conceitos das estruturas aditivas, das estruturas multiplicativas, das relações número-espaço e da álgebra (VERGNAUD, 1993). Seu foco não está apenas na construção de conceitos matemáticos. De acordo com Fioreze, Barone e Basso (2008, p.3), “o objetivo desta teoria é fornecer uma estrutura explicativa às pesquisas sobre atividades cognitivas complexas, em especial às relacionadas às aprendizagens científicas e técnicas". É uma estrutura consistente, que se apoia nas representações, nos esquemas e conceitos utilizados pelos alunos para resolver problemas em diversas áreas. Podemos afirmar que, durante atividades de programação, o estudante está em constante ação e é levado a resolver situações variadas que lhe permitem combinar e descobrir diferentes aspectos dos conceitos envolvidos. Assim, essa teoria se apresenta como uma ferramenta para auxiliar o professor na compreensão desses processos e na proposição de novas situações que auxiliem o aluno na construção de conceitos via programação.

As teorias gerais de desenvolvimento não são tão próximas dos conteúdos escolares como a teoria proposta por Vergnaud, e isto é o que a torna relevante para o ambiente de ensino (VERGNAUD, 2011). Essa visão não afasta a Teoria dos Campos Conceituais das descobertas das demais teorias; ela as considera e utiliza como base, como é o caso dos conceitos de assimilação, acomodação e esquema propostos por Jean Piaget em sua Teoria Psicogenética (CHIAROTTINO, 2005). Na perspectiva de melhor compreender essa relação, apresentamos uma exposição desses conceitos piagetianos que dão suporte à Teoria dos Campos Conceituais relacionando-os com as noções matemáticas.

A inteligência, para Piaget, é "a adaptação às situações novas e é então uma construção contínua das estruturas." (BRINGUIER, 1993, p. 61). Essa adaptação representa um equilíbrio, que nunca é perfeito, entre os esquemas de assimilação e acomodação. Um esquema representa "aquilo que é generalizável numa determinada 
ação" (CHIAROTTINO, 2005, p. 18). Por exemplo, o esquema de ângulo corresponde a identificar um giro independente do objeto que gira ou quanto ele girou. A partir desses esquemas, o estudante faz assimilações, num processo em que busca incorporar o objeto às suas estruturas, sem que haja mudanças no pensamento. Já a acomodação é um processo de ajuste do esquema a uma situação particular, e durante esse processo há mudanças no modo de pensar e agir do estudante. Diante dessas definições, Piaget considera que os processos de assimilação e acomodação são complementares e indissociáveis (BRINGUIER, 1993). Voltando ao exemplo anterior do esquema de ângulo, ao programar o movimento de um sprite no Scratch que está em uma determinada posição inicial, o indivíduo pode assimilar o comando "gire" ao seu esquema de ângulo, mesmo que não identifique o que representam os valores ali acrescentados. Porém, no momento em que ele faz experimentações de valores e observa seus efeitos gráficos, está em um processo de busca pelo equilíbrio, ou seja, de adaptação do seu esquema anterior. Ao final, quando passa, por exemplo, a inserir o valor de $90^{\circ}$ com o intuito de que o sprite faça o movimento de um quarto de uma volta, estará demonstrando que seu esquema inicial foi acomodado, modificado para atender às novas situações que lhe foram apresentadas pelo programa.

As contribuições de Piaget sobre o processo de aprendizagem a partir da assimilação e acomodação dos esquemas dão suporte para que Vergnaud proponha "uma estrutura que permita compreender as filiações e rupturas entre conhecimentos, em crianças e adolescentes, entendendo-se por 'conhecimento' tanto as habilidades quanto as informações expressas” (VERGNAUD, 1993, p. 1). Nesse sentido, considera que o processo de aprendizagem é longo e se desenvolve a partir das diferentes situações que ocorrem dentro e fora da escola. Essas situações levam o aluno a passar por etapas que envolvem filiações, pois os novos conhecimentos apoiam-se em conhecimentos anteriores, e rupturas, pois, em alguns momentos, é necessário abandonar ideias e ações anteriores para a construção de novas competências (VERGNAUD, 2011).

\section{AS SITUAÇÕES}

Uma situação consiste em uma tarefa, que pode ser prática ou teórica, que leve o estudante a traçar relações, explorar, criar hipóteses e verificá-las, a fim de obter uma solução. Desta forma, a Teoria dos Campos Conceituais não reduz um conceito à sua definição, mas defende que "é através das situações e dos problemas a resolver que um conceito adquire sentido para a criança" (VERGNAUD, 1993, p. 1). O sentido que permite ao estudante compreender um conceito refere-se aos esquemas que são despertados a partir de uma situação. Além disso, "toda situação complexa é uma combinação de situações elementares, e não se pode contornar a análise das tarefas cognitivas que podem ser geradas por elas" (VERGNAUD, 1993, p. 17). Com isso Vergnaud indica que analisar uma situação mais complexa a partir de subtarefas auxilia na sua compreensão. Esse tipo de ação é frequentemente utilizado durante atividades de programação; a montagem de um programa mais complexo pode ser dividida em subprogramas que, unidos, desempenham a função desejada, ou ainda, a análise de um bug $^{1}$ pode ser feita separando-o em partes a fim de verificar etapas não condizentes com o resultado esperado.

Existem dois grupos de situações que se distinguem pelas competências que o aluno possui para lidar com elas. A primeira é a classe das situações em que o estudante

\footnotetext{
${ }^{1}$ Erro na programação.
} 
já possui as competências necessárias para lidar com elas, apresentando um comportamento automatizado, organizado por apenas um esquema; e a segunda, a classe das situações em que o estudante não possui todas as competências necessárias, utilizando vários esquemas para chegar à solução desejada, e, para isso, esses esquemas precisam ser "acomodados, descombinados e recombinados"(VERGNAUD, 1993, p. 2).

\section{ESQUEMA}

O conceito de esquema criado por Piaget é complementado por Vergnaud quando o define como

[...] a organização invariante do comportamento para uma classe de situações dada. É nos esquemas que se devem pesquisar os conhecimentos-em-ação do estudante, isto é, os elementos cognitivos que fazem com que a ação do estudante seja operatória. (VERGNAUD, 1993, p. 2).

Portanto, para o autor, um esquema é um conjunto de ações, de coleta de dados e controle que variam de acordo com cada situação, organizando as ações e o pensamento. Um programa criado no Scratch é um exemplo de esquema; ele demonstra a ação do estudante para a resolução de uma situação e pode ser empregado em situações da mesma classe.

Voltando às classes de situações já citadas, podemos afirmar que os esquemas se enquadram perfeitamente na primeira classe de situações. Já na segunda classe também há uma estruturação em esquemas. Porém, como não há um esquema específico para aquela situação, o estudante busca em seu repertório esquemas que tenham alguma afinidade com a situação dada. Neste caso, o esquema pode não ser efetivo, sendo necessária modificação, combinação de esquemas ou acréscimo de elementos cognitivos, criando novos esquemas.

Um esquema é composto por: invariantes operatórias (são os conceitos-em-ação e teoremas-em-ação); metas e antecipações (são as possíveis direções e finalidades da atividade, efeitos esperados); regras de ação do tipo "se...então" (permitem ao estudante criar a sequência de ações); inferências (ou raciocínio - permitem ao estudante raciocinar durante a situação a partir das informações dos outros três componentes do esquema).

Um esquema está sempre apoiado em uma conceitualização implícita. Mesmo que o estudante não saiba expressar esse conceito, ele precisa compreendê-lo para que seu esquema funcione corretamente. Um exemplo pode ser representado pelo movimento de um sprite no Scratch considerando suas coordenadas cartesianas. A figura 3 ilustra o sprite em sua posição inicial.

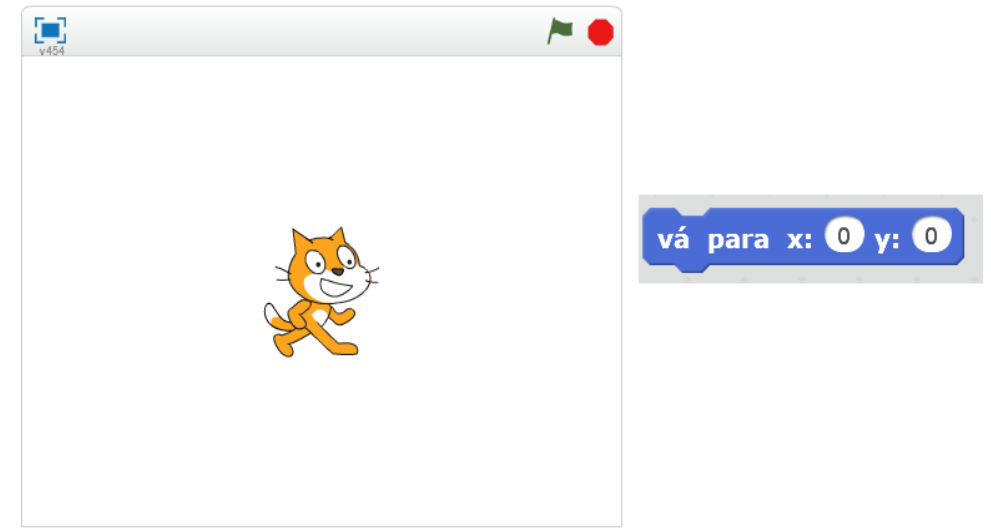

Figura 3 - Sprite na sua posição inicial Fonte: acervo da primeira autora 
O sprite está inicialmente no centro da tela (posição $\mathrm{x}=0$ e $\mathrm{y}=0$ ). Para deslocá-lo horizontalmente usando o comando "vá para $\mathrm{x}$ : ", é necessário que o estudante compreenda o sistema de coordenadas identificando que os valores de $\mathrm{x}$ correspondem a esse deslocamento; ou seja, mantendo-se o valor de y constante e alterando os valores de $\mathrm{x}$, o sprite fará deslocamentos horizontais na tela. Mesmo que o estudante não consiga expressar o que é um sistema de coordenadas cartesianas, ele compreende que, alterando os valores de x, obtém o deslocamento horizontal, ou seja, esse conhecimento ainda está implícito na ação.

Os conhecimentos contidos num esquema são denominados conceitos-em-ação e teoremas-em-ação. O uso do termo "em ação" por Vergnaud, como o nome se refere, corresponde à ideia de que o estudante usa esses conhecimentos durante a situação. Os conceitos-em-ação são uma categoria de pensamento, não sendo necessariamente conceitos científicos; são eles que permitem ao estudante verificar se uma determinada informação é ou não pertinente para enfrentar uma situação, ou seja, constituem-se em conceitos mobilizados pelo estudante para pensar na situação.

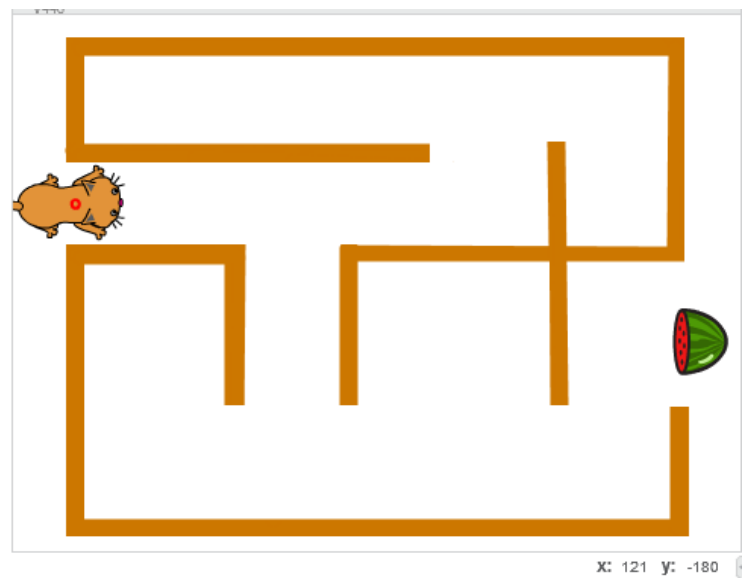

Figura 4 - Labirinto: conceitos ativados

Fonte: acervo da primeira autora

A situação de programar o sprite gato para que ele percorra o labirinto (figura 4) até a melancia pode levar o estudante a mobilizar conceitos como ângulo, deslocamento, paralelismo, ângulo reto, plano cartesiano, algoritmo e programação linear. Esses são possíveis conceitos a serem utilizados para a resolução desse problema. Um estudante que possui poucas experiências com programação e com os conceitos envolvidos, poderá realizar uma programação mais exploratória testando comandos e criando um algoritmo que representa cada etapa do caminho a ser percorrido pelo sprite, colocando em ação os conceitos de deslocamento, ângulo, programação linear e algoritmo. A partir dessa situação, identificamos a existência de vários conceitos envolvidos durante a sua solução, mas cada estudante, a partir de seus esquemas e experiências anteriores, pode ativar um grupo diferente de conceitos para solucioná-la.

Partindo de situação similar a representada pela figura 4, podemos pensar sobre a presença de teoremas-em-ação. Um aluno, durante a resolução do problema, poderia fazer suposições como: para deslocar o sprite é necessário utilizar apenas o bloco de movimento; o sprite gira a partir da posição anterior ao momento do giro; o deslocamento horizontal pode ser obtido alterando o valor da variável x; a programação precisa ser estruturada na sequência em que se deseja produzir os movimentos. Diante dos exemplos anteriores, destacamos que os teoremas-em-ação representam uma proposição que pode ser verdadeira ou não. Esses teoremas não são necessariamente 
teoremas científicos, mas suposições, propriedades que os alunos atribuem aos conceitos durante a busca pela solução para uma situação.

Os conceitos-em-ação estão diretamente ligados aos teoremas-em-ação. Uma proposição só pode ser formulada se possui um conceito a ela associado, e o contrário também se verifica: não há conceitos-em-ação sem teoremas-em-ação. Os conceitos só são conceitos porque resultaram de proposições que, durante a ação, foram constatadas como verdadeiras, tornando-se conceitos.

Os conceitos-em-ação e os teoremas-em-ação recebem essa denominação por expressarem conhecimentos e teoremas que não são estáticos e acabados, mas estão em ação, são os frutos colhidos de experiências anteriores e que são utilizados como válidos em situações semelhantes. Ao resolver um problema, o aluno busca a solução em problemas similares já resolvidos para que possa utilizar esquemas conhecidos e, a partir de algumas avaliações prévias, modificá-los, alterá-los, descobrindo outros aspectos ou até mesmo novos esquemas. Com o tempo, alguns desses esquemas se tornam automatizados pelos alunos, e, segundo Vergnaud (1993), podem indicar possíveis compreensões e relações estabelecidas pelo estudante.

\section{CAMPO CONCEITUAL E CONCEITO}

Vergnaud considera que o conhecimento está organizado em campos conceituais, que são definidos "como um conjunto de situações cujo domínio requer uma variedade de conceitos, de procedimentos e de representações simbólicas em estreita conexão" (VERGNAUD, 1986, p. 84). Neste sentido, o autor propõe o estudo de um campo em vez de um conceito, pois resolver uma situação qualquer exige a união de vários conceitos. Deslocar um sprite no Scratch representa um exemplo de diversos conceitos interligados: giro, movimento, plano cartesiano, programação e sequência.

Um conceito é uma síntese do conjunto das situações que constituem a referência de suas diversas propriedades e do conjunto dos esquemas que são utilizados pelo estudante (VERGNAUD, 1993). Esta definição é chamada pelo autor de uma definição pragmática, em que ele apresenta de forma completa todos os elementos que permitem ao estudante construir de fato um conceito. Ao identificar a operacionalidade de um conceito, não se pode considerar apenas a ação operatória, mas é necessário analisar o uso de significantes; ou seja, o indivíduo deve ser capaz de expressar o conceito de forma simbólica, através de uma linguagem natural, símbolos, representações, diagramas, entre outros. Diante disso, Vergnaud (CARVALHO JR.; AGUIAR JR., 2008; MAGINA, 2005; VERGNAUD, 1993, p. 8) define conceito como uma trinca de conjuntos $C=(S, I, R)$, sendo:

\footnotetext{
S - conjunto das situações que dão sentido ao conceito (referência)

I - conjunto das invariantes em que se baseia a operacionalidade dos esquemas (significado)

$\mathrm{R}$ - conjunto das formas de linguagem (ou não) que permitem representar simbolicamente o conceito, suas propriedades, as situações e os procedimentos de tratamento (significante).
}

A construção de um conceito se dá a partir da relação entre esses três conjuntos; ou seja, para compreender um conceito, um aluno precisa de situações em que este conceito esteja inserido, esquemas que lhe permitam buscar soluções (significado) e diferentes formas de representação (significante). 
Considerando o conceito de ângulo via programação, podemos supor as seguintes situações, invariantes e representações que formam a trinca desse conceito expostas no quadro 1 .

Quadro 1 - Trinca S, I, R do conceito de ângulo

\begin{tabular}{|c|c|c|}
\hline Conjunto das Situações - S & Conjunto das Invariantes - I & Conjunto das Representações - R \\
\hline $\begin{array}{l}\text { - Movimentos e giros } \\
\text { realizados no skate } \\
\text { - Construção de figuras } \\
\text { - Movimentos do sprite no } \\
\text { Scratch } \\
\text { - Medida de um giro } \\
\text { - Construção de comandos } \\
\text { para um jogo }\end{array}$ & $\begin{array}{l}\text { - Uma volta completa é } 360^{\circ} \\
\text { - O valor do grau indica o giro } \\
\text { dado } \\
\text { - O giro do sprite se dá a partir da } \\
\text { sua posição } \\
\text { - O giro de } 90^{\circ} \text { representa um } \\
\text { canto } \\
\text { - O sprite pode ter seu giro } \\
\text { revertido através de um giro de } \\
\text { mesmo valor, porém em sentido } \\
\text { contrário ao dado }\end{array}$ & $\begin{array}{l}\text { - gestos que expressam o giro } \\
-\quad \text { movimentos com o corpo } \\
\text { colocando-se na posição do sprite } \\
\text { para imitá-lo }\end{array}$ \\
\hline
\end{tabular}

A construção de conceitos ocorre a partir das situações que o estudante já domina; por isso podemos afirmar que ela tem características locais, e o domínio do estudante está restrito aos seus esquemas que precisam ser ampliados. Vergnaud (2011; 1993), argumenta que essa construção não é imediata a partir de uma explicação, mas um processo lento no qual novas situações devem ser constantemente introduzidas, cada vez mais complexas, ampliando o repertório de esquemas. Se um aluno tem uma concepção errônea, ela só pode ser mudada se confrontada com situações em que não pode ser utilizada. Programar movimentos de um sprite é algo constante em projetos no Scratch, como, por exemplo, montar um algoritmo em que o sprite faça um determinado deslocamento, criar um programa em que, ao acionar uma tecla do teclado, o sprite aponta para uma direção e se move, criar um programa que desenha polígonos. Esses exemplos de movimentos utilizam o conceito de ângulo sob diferentes aspectos, que variam de acordo com a situação e permitem ao aluno explorar e testar seus esquemas para a construção do conceito.

\section{PAPEL DO PROFESSOR}

O papel do professor é de auxiliar o aluno na construção dos conceitos; por isso,

Um bom desempenho didático baseia-se necessariamente no conhecimento da dificuldade relativa das tarefas cognitivas, dos obstáculos habitualmente enfrentados, do repertório de procedimentos disponíveis e das representações possíveis. (VERGNAUD, 1993, p. 17).

Nesse sentido, cabe ao professor conhecer o aluno, observá-lo, procurando identificar os conhecimentos explícitos, quais ainda estão implícitos, reconhecer os esquemas utilizados, pesquisar as afinidades e rupturas que ele realiza a partir de um conjunto de situações. Observar apenas o resultado final não leva o professor à compreensão do processo pelo qual o aluno passou para chegar ao resultado, tampouco revela os esquemas por ele utilizados, e isso dificulta a proposição de situações que o auxiliem na construção de conceitos.

A proposição de exercícios de caráter repetitivo, considerando o ensino como uma forma de aquisição de conhecimento por condicionamento, por hábito, é o que Vergnaud denomina de erro pedagógico, pois, segundo o autor, o aluno adquire regras 
que precisam ser aplicadas e testadas em diferentes situações (VERGNAUD, 2009). Elas só são solidificadas a partir da compreensão, no momento em que o aluno faz ligações. Diante disso, a proposição de situações variadas, o acompanhamento contínuo, a discussão a respeito dos caminhos percorridos contribui para o processo de repensar os procedimentos tomados. O fato de analisar um bug na programação em vez de ignorá-lo e recomeçar um novo programa é uma forma de compreender a situação, fazer inferências, antecipar novos resultados, o que leva a contribuir para além do acerto, para uma mudança de pensamento.

O professor pode ser um mediador, não apenas no sentido de acompanhar a aprendizagem e de propor situações aos alunos, mas também de propor representações simbólicas acessíveis que colaborem para a formação da estrutura conceitual. Neste caso, há a necessidade de conhecer o campo conceitual a ser estudado, possíveis representações, esquemas a serem utilizados, a fim de melhor compreender e auxiliar o aluno em seu processo de descoberta.

\section{CONSIDERAÇÕES FINAIS}

A Teoria dos Campos Conceituais permite um olhar direcionado para a compreensão de conceitos pelos estudantes e, em particular, conceitos matemáticos. Ao se defrontar com diferentes situações sobre um mesmo conceito, os estudantes têm a oportunidade de testar hipóteses, descobrir possibilidades, realizar inferências, combinando e recombinando seus esquemas contribuindo para a aprendizagem de matemática. Essa compreensão dos conceitos permite utilizá-los e representá-los, sendo ponto de partida para a construção de novos saberes.

O Scratch apresenta-se como uma ferramenta acessível, com uma linguagem simples e visual, o que permite que jovens estudantes construam programas para resolver problemas. As atividades de programação em Scratch permitem explorar uma variedade de situações e de testagens de teorias pelos próprios estudantes. Nesse sentido, ao observar atividades em Scratch, identificamos a contribuição dessa ferramenta na expressão de pensamentos matemáticos, auxiliando na construção de conceitos e o desenvolvimento do pensamento computacional.

Ao utilizar a Teoria dos Campos Conceituais como base para a elaboração de atividades de programação e de análise de produções em Scratch, consideramos a possibilidade dessa teoria auxiliar na compreensão dos esquemas elaborados pelos alunos ao criarem programas em Scratch. Nesse ato de programar, as expressões e falas dos alunos podem revelar seus teoremas-em-ação e conceitos-em-ação. A presença e identificação desses elementos, por sua vez, permitem ao professor projetar sua prática de modo a oferecer aos estudantes novas possibilidades de atividades que apoiem o desenvolvimento da trinca situações-invariantes-representações, necessária para a formação de um conceito e que contribuam em processos de aprendizagem.

\section{REFERÊNCIAS}

BRINGUIER, J. C. Conversando com Piaget. $2^{a}$ Ed. Tradução: Maria José Guedes. Rio de Janeiro: Bertrand, 1993. 210p

CARVALHO JR., G. D.; AGUIAR JR., O. Os Campos Conceituais de Vergnaud como ferramenta para o planejamento didático. Caderno Brasileiro de Ensino de Física, v.25, n.2, p.207-227, 2008. 
CHIAROTTINO, Z. P. Os "estágios" do desenvolvimento da inteligência. Revista Viver: mente e cérebro. Coleção Memória da Pedagogia: Jean Piaget, Ediouro: Rio de Janeiro, n.1, p.16-23, 2005.

FIOREZE, L. A.; BARONE, D.; BASSO, M. Atividades Digitais, a Teoria dos Campos Conceituais e o desenvolvimento dos conceitos de proporcionalidade. Revista Novas Tecnologias na Educação - UFRGS. Porto alegre. v.6, n. 2, 10 p., 2008. Disponível em: < http://seer.ufrgs.br/index.php/renote/article/view/14691/8600>. Acesso em: 16 ago. 2017.

MAGINA, S. A. A Teoria dos Campos Conceituais: contribuições da Psicologia para a prática docente. In. Encontro Regional de Professores de Matemática, 18., 2005, São Paulo: Unicamp. Disponível em: <http://www.ime.unicamp.br/erpm2005/anais/conf/conf_01.pdf>. Acesso em: 04 mar. 2017.

PAPERT, S. Logo: computadores e educação. Tradução: José Armando Valente. São Paulo: Brasiliense, 1985. 253 p.

RESNICK, M. et al. Scratch: Programming for all. Communications of the ACM. [s.l.]. $\mathrm{n}$ 11, p. 60-67, nov. 2009. Disponível em $<$ http://web.media.mit.edu/ mres/papers/Scratch-CACM-final.pdf >. Acesso em: 20 maio 2015.

ROCHA, K. C. Programação em Scratch na sala de aula de Matemática: investigações sobre a construção do conceito de ângulo. 2017. 211 f. Dissertação (Mestrado Profissional em Ensino de Matemática) - Programa de Pós-Graduação em Ensino de Matemática, Instituto de Matemática e Estatística, Universidade Federal do Rio Grande do Sul, Porto Alegre, 2017.

VERGNAUD, G. Psicologia do desenvolvimento cognitivo e didática das matemáticas. Um exemplo: as estruturas aditivas. Análise Psicológica, v. 1, p.75-90, 1986.

Teoria dos campos conceituais. In Nasser, L. (Ed.) Anais do $1^{\circ}$ Seminário Internacional de Educação Matemática do Rio de Janeiro. 1993. p. 1-26.

A criança, a matemática e a realidade: problemas do ensino da matemática na escola elementar. Tradução: Maria Lúcia Faria Moro. Curitiba: UFPR, 2009. 322p.

O longo e o curto prazo na aprendizagem da matemática. Educar em Revista, n. Especial 1/2011, Editora UFPR: Curitiba, p.15-27, 2011.

VITALE, B. Computador na escola: um brinquedo a mais? Ciência Hoje, v. 13, n. 77, p. 19-25, out./nov. 1991.

WING, J. M. Computational Thinking. Communications of the ACM, vol. 49, n. 3, p.33-35, mar 2006. 